\title{
SIMULAÇÃO E PROJETO DE UMA UNIDADE DE PRODUÇÃO DE BIODIESEL VIA CATÁLISE HETEROGÊNEA A PARTIR DO ÓLEO DE DENDÊ
}

\author{
C. B. Sousa, V. A. M. Gomes, S. M. P. Lucena \\ Universidade Federal do Ceará, Departamento de Engenharia Química \\ E-mail para contato: caiobragasousa@gmail.com
}

\begin{abstract}
RESUMO - Uma unidade de produção contínua de biodiesel com carga de 8000 $\mathrm{kg} / \mathrm{h}$ de óleo de dendê e metanol, com o uso a rota de transesterificação heterogênea, foi simulada e projetada com uso do software Aspen HYSYS 7.3. O pacote termodinâmico NRTL foi empregado para representar as propriedades. Um reator de leito fixo foi operado a $64,8^{\circ} \mathrm{C}$ com razão álcool/óleo de 17,1 . Uma coluna de destilação a vácuo foi projetada para reciclar $99 \%$ do metanol em excesso. Após a separação de fases, o biodiesel foi enviado para uma segunda coluna de destilação a vácuo e a fase glicerina para um tanque flash também a vácuo. O biodiesel obtido da coluna teve uma pureza de $99,9 \% \mathrm{~m} / \mathrm{m}$, em acordo com as especificações da ANP. Além disso a corrente de saída de glicerina possuiu uma pureza de $98,7 \% \mathrm{~m} / \mathrm{m}$, com maior valor agregado que o obtido pela rota homogênea.
\end{abstract}

\section{INTRODUÇÃO}

Por motivos econômicos, ambientais e geopolíticos, diversos países têm concentrado suas atenções em termos de pesquisa científica no intuito de alterarem suas matrizes energéticas, que em nível global é fortemente dependente dos combustíveis fósseis: petróleo, gás natural e carvão mineral. Tal atenção tem-se voltado principalmente para as fontes renováveis de energia, que diferentemente dos combustíveis fósseis, não estão atreladas a prazos de esgotamento. Dentre as diversas opções de energias renováveis, tem ganho destaque o biodiesel, um promissor substituto do óleo diesel derivado de petróleo (Lee et al., 2011).

O biodiesel é produzido a partir de uma reação de transesterificação, geralmente em meio catalítico, onde moléculas de triacilglicerídeos presentes em óleos vegetais e gorduras reagem com um álcool de cadeia curta, em geral metanol e etanol, para produzirem monoalquil ésteres. A Figura 1, adaptada de West et al., (2008), mostra a reação de transesterificação.

$$
\begin{aligned}
& \mathrm{CH}_{2}-\mathrm{OOC}-\mathrm{R}_{1} \quad \mathrm{R}_{1}-\mathrm{COO}-\mathrm{R}^{\prime} \quad \mathrm{CH}_{2}-\mathrm{OH} \\
& \text { I Cat. I } \\
& \mathrm{CH}-\mathrm{OOC}-\mathrm{R}_{2}+3 \mathrm{R}^{\prime} \mathrm{OH} \leftrightarrow \mathrm{R}_{2}-\mathrm{COO}-\mathrm{R}^{\prime}+\mathrm{CH}-\mathrm{OH} \\
& \begin{array}{lll}
\mathrm{CH}_{2}-\mathrm{OOC}-\mathrm{R}_{3} & \mathrm{R}_{3}-\mathrm{COO}-\mathrm{R}^{\prime} & \mathrm{CH}_{2}-\mathrm{OH}
\end{array}
\end{aligned}
$$

Figura 1 - Reação de transesterificação. 
O processo convencional de produção de biodiesel, responsável pela maior parte da produção atualmente, é a transesterificação de óleos vegetais refinados usando metanol como álcool de cadeia curta e um catalisador alcalino homogêneo, principalmente o $\mathrm{NaOH}$ (Di Serio et al., 2008). Tal processo é conhecido como rota homogênea básica, ou simplesmente rota homogênea.

Embora a transesterificação pela rota homogênea básica conduza a altas conversões do óleo vegetal em mono-alquil ésteres, esta apresenta uma séria de desvantagens: é energointensiva; o catalisador tem que ser removido do efluente do reator, o que implica o uso de processos de purificação complexos, com a produção de consideráveis volumes de água residual; ácidos graxos livres presentes óleo interferem no processo, em virtude da ocorrência de reações competitivas de saponificação (Atadashi et al., 2011).

Outro problema comum referente a transesterificação homogênea está na utilização de óleo vegetal refinado, sendo que o custo da matéria prima graxa representa cerca $75 \%$ dos custos totais de produção (Morais, 2010). No cenário brasileiro, a maioria das plantas operam com óleo de soja, o que causa um déficit econômico, sendo este suprido somente por subsídios governamentais. No entanto, a diversificação das matérias primas pode ser uma saída para o impasse, aja visto que algumas fontes de matéria prima, como o óleo de dendê, apresentam grandes disponibilidades de óleo. A palmeira de dendê pode chegar a níveis de produção de até 6 toneladas/hectare. No entanto, o principal problema referente ao óleo de dendê refere-se a sua acidez, que em geral é elevada, fato que inviabiliza o seu uso pela rota homogênea (Santana, 2008).

Neste contexto, a transesterificação usando catalisadores heterogêneos, ou rota heterogênea, apresenta vantagens sobre a rota homogênea. A mais importante delas refere-se a exclusão de seguidas etapas de purificação, que envolvem a neutralização do meio reacional, lavagem e secagem do biodiesel, que na catálise homogênea são etapas fundamentais. Como consequência, há uma redução no número de equipamentos de processo, redução na quantidade de água requerida, redução na quantidade de efluentes com custos ambientais reduzidos, e corrosão em equipamentos (XIAO et al., 2012). Outra importante vantagem da rota heterogênea diz respeito a sua insensibilidade em relação a acidez do óleo, o que torna esta rota ideal para a transesterificação do óleo de dendê em biodiesel. Além disso o processo também produz uma glicerina com elevada pureza, com pelo menos $98 \% \mathrm{~m} / \mathrm{m}$, sendo que esta possui um maior valor agregado (Di Serio et al., 2008).

Até o momento, existem poucos trabalhos publicados sobre simulações de plantas de processo usando a rota heterogênea na produção de biodiesel. $\mathrm{O}$ trabalho de West et al. (2009) propõe um modelo de processo no software Aspen HYSYS. Entretanto, tal modelo tem por inconveniente o uso de um reator de conversão, um módulo de cálculo contido na maioria dos simuladores de processo, sendo utilizado somente como uma primeira aproximação em cálculos de projeto. O reator de conversão realiza cálculos de reações químicas baseados somente na estequiometria das reações (Towler; Sinnot, 2008). Em simulações de processo mais realísticas, reatores do tipo Tanque Perfeitamente Agitado (CSTR), Reatores Tubulares (PFR) ou Reatores de Leito Fixo (PBR) devem ser utilizados. No entanto, o uso destes reatores requer obrigatoriamente a disponibilidade de dados cinéticos das reações de interesse. 
O objetivo deste trabalho foi o de simular e projetar uma unidade contínua de biodiesel com o uso da rota de transesterificação heterogênea no Aspen HYSYS 7.3. Um Reator de Leito Fixo (PBR) foi escolhido para representar o reator industrial, onde os dados cinéticos e de constantes de equilíbrio do modelo de Eley-Rideal para o catalisador foram obtidos do trabalho de Xiao et al., (2010). Posteriormente, os equipamentos de processo foram projetados utilizando-se de ferramentas implementados no próprio software.

\section{DADOS ENTRADA PARA A SIMULAÇÃO}

Inicialmente foram definidos os componentes químicos participantes da simulação no Simulation Basis Environment (Ambiente Básico de Simulação) do Aspen HYSYS 7.3. Os componentes glicerol $\left(\mathrm{C}_{3} \mathrm{H}_{8} \mathrm{O}_{3}\right)$, metanol $\left(\mathrm{CH}_{4} \mathrm{O}\right)$ e palmitato de metila $\left(\mathrm{C}_{17} \mathrm{H}_{34} \mathrm{O}_{2}\right)$ para representar o biodiesel já se encontravam na base de dados do software. Entretanto, o óleo de dendê não constava na base de dados, sendo que este foi representado como um pseudocomponente do triglicerídeo tripalmitina $\left(\mathrm{C}_{51} \mathrm{H}_{98} \mathrm{O}_{6}\right)$. O pseudocomponente foi construído e suas propriedades termodinâmicas foram calculadas com uso de um método de contribuição de grupos do Aspen HYSYS 7.3 (Aspen HYSYS, 2011). Os dados de entrada para os cálculos foram a massa molar da tripalmitina $M$, a distribuição dos grupos e o ponto normal de ebulição $T_{b}$, sendo este último obtido do trabalho de Lee et al., (2011). A Tabela 1 resume esses dados de entrada. O modelo de NRTL foi escolhido para representar o comportamento da fase líquida, em virtude da presença de componentes altamente polares, e o método UNIFAC LLE foi utilizado para calcular os parâmetros de interação binária em falta no banco de dados do software (Lee et al., 2011).

Tabela 1 - Dados de entrada para a simulação

\begin{tabular}{|c|c|}
\hline Dados da Tripalmitina & Valores \\
\hline$M(\mathrm{~g} / \mathrm{mol})$ : massa molar & 807,32 \\
\hline$T_{b}\left({ }^{\circ} \mathrm{C}\right)$ : temperatura nomal de ebulição ${ }^{\mathrm{a}}$ & 409,3 \\
\hline \multicolumn{2}{|l|}{ Dados Cinéticos e de Constantes de Equilíbrio } \\
\hline 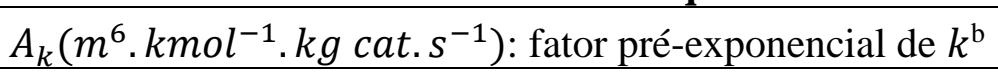 & $8,69 \times 10^{15}$ \\
\hline$E_{k}(J / m o l)$ : energia de ativação de $k^{\mathrm{c}}$ & 112923 \\
\hline$A_{B}\left(\mathrm{~m}^{3} \cdot \mathrm{kmol}^{-1}\right)$ : fator pré-exponencial de $K_{B}{ }^{\mathrm{d}}$ & $7,63 \times 10^{-07}$ \\
\hline$E_{B}(J / m o l):$ energia de ativação de $K_{B}{ }^{\mathrm{e}}$ & $-37536,6$ \\
\hline$A_{C}\left(m^{3} \cdot k m o l^{-1}\right)$ : fator pré-exponencial de $K_{C}{ }^{\mathrm{f}}$ & $3,27 \times 10^{-10}$ \\
\hline$E_{C}(\mathrm{~J} / \mathrm{mol})$ : energia de ativação de $K_{C}^{\mathrm{g}}$ & $-61238,9$ \\
\hline$K$ : constante de equilíbrio a $64,8^{\circ} \mathrm{C}^{\mathrm{h}}$ & 0,289 \\
\hline \multicolumn{2}{|l|}{ Parâmetro do Leito Reacional } \\
\hline$\rho_{p}(\mathrm{~kg} / \mathrm{m} 3):$ massa específica das partículas ${ }^{\mathrm{i}}$ & 2306,4 \\
\hline$\varepsilon:$ porosidade do leito $^{\mathrm{j}}$ & 0,52 \\
\hline
\end{tabular}


Em seguida a cinética da reação foi caracterizada com o uso do modelo de Eley-Rideal, representado pela Equação 1 seguinte, e dados cinéticos e de constantes de equilíbrio experimentais do trabalho de Xiao et al., (2010) para o catalisador heterogêneo hidrotalcita $\mathrm{KF} / \mathrm{Ca}-\mathrm{Mg}-\mathrm{Al}$, resumidos na Tabela 1 .

$$
r_{p}=\frac{k\left(C_{A} C_{B}-\frac{1}{K} \frac{C_{C}^{3} C_{D}}{C_{B}^{2}}\right)}{1+K_{B} C_{B}+K_{C} C_{C}}
$$

Onde $C_{A}, C_{B}, C_{C}$ e $C_{D}$ são as concentrações molares de óleo, metanol, glicerol e biodiesel respectivamente em kmol. $\mathrm{m}^{-3}, \quad k$ a constante de velocidade em

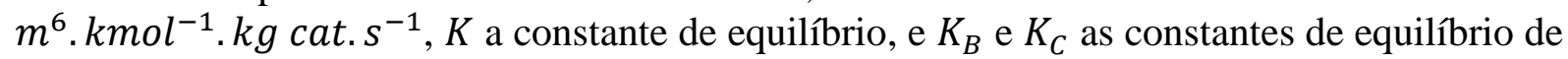
adsorção no catalisador para o metanol e o glicerol em $\mathrm{m}^{3} \cdot \mathrm{kmol}^{-1}$, respectivamente.

Para o modelo do reator, os parâmetros do leito foram obtidos do trabalho Xiao et al., (2012), sendo estes a massa específica das partículas $\rho_{p}$ e a porosidade do $\varepsilon$, mostradas na Tabela 1.

\section{METODOLOGIAS DE PROJETO}

O Reator de Leito Fixo (PBR) foi projetado para uma conversão global de 97,5\%, com as estimativas de seu diâmetro $D_{r}$, comprimento $L_{r}$ em metros (m) e massa de catalisador $m_{c}$ em kg. Um trocador de calor casco e tubos para aquecimento foi dimensionado de acordo com o Standard Method (Metodolodia Padrão) implementada no software para obtenção o diâmetro do casco $\phi_{c}$, diâmetro dos tubos $\phi_{t}$, número de tubos $N_{t}$ e comprimento dos tubos $L_{t}$, sendo todas estas medidas em metros. As colunas de destilação foram projetadas de acordo com as correlações de cálculo do diâmetro da torre $D_{t}$, espaçamento entre os pratos $T S$ e número de válvula por prato $N_{v}$. O tanque flash V-101 foi projeto pelo cálculo do diâmetro $D_{f}$ e a altura $H_{f}$ em metros (Aspen HYSYS, 2011). O decantador V-100 para separar as fases biodiesel e glicerina foi projetado de acordo com a seguinte equação para o cálculo do tempo de residência $t$ em horas necessário para separação de fases (McCabe et al., 2005):

$$
t=\frac{100 \mu}{\rho_{A}-\rho_{B}}
$$

Onde $\rho_{A}$ e $\rho_{B}$ são as massas específicas das fases biodiesel e glicerina respectivamente em $\mathrm{kg} \cdot \mathrm{m}^{-3}$, e $\mu$ a viscosidade dinâmica da alimentação do decantador em cP. Com o valor de $t$ foi possível obter o volume em metros cúbicos. Por fim o diâmetro $D_{d}$ em metros foi obtido como sendo cindo vezes o comprimento $L_{d}$ (McCabe et al., 2005).

Todas as bombas foram dimensionadas pelo cálculo da potência necessária $W_{b}$ levando em consideração uma eficiência de $75 \%$. 


\section{RESULTADOS E DISCUSSÕES}

A unidade foi construída no software com uma base de cálculo de $8000 \mathrm{~kg} / \mathrm{h}$ de óleo dendê e metanol, na razão molar álcool/óleo de 17,1, onde a Figura 1 mostra um Diagrama de Fluxo de Processo (PFD) construído a partir dos resultados da simulação. As correntes Óleo e Metanol foram bombeadas separadamente nas bombas centrífugas P-100 e P101. Estas correntes foram misturas no mixer estático MIX-100, sendo este um equipamento adequado para realizar misturas espécies que tendem a formar sistemas bifásicos, como é o caso de óleos vegetais e álcoois. A corrente 3 formada no mixer foi então aquecida no trocador de calor casco e tubos E-100 até a temperatura de reação. A reação ocorreu no reator PFR-100 de

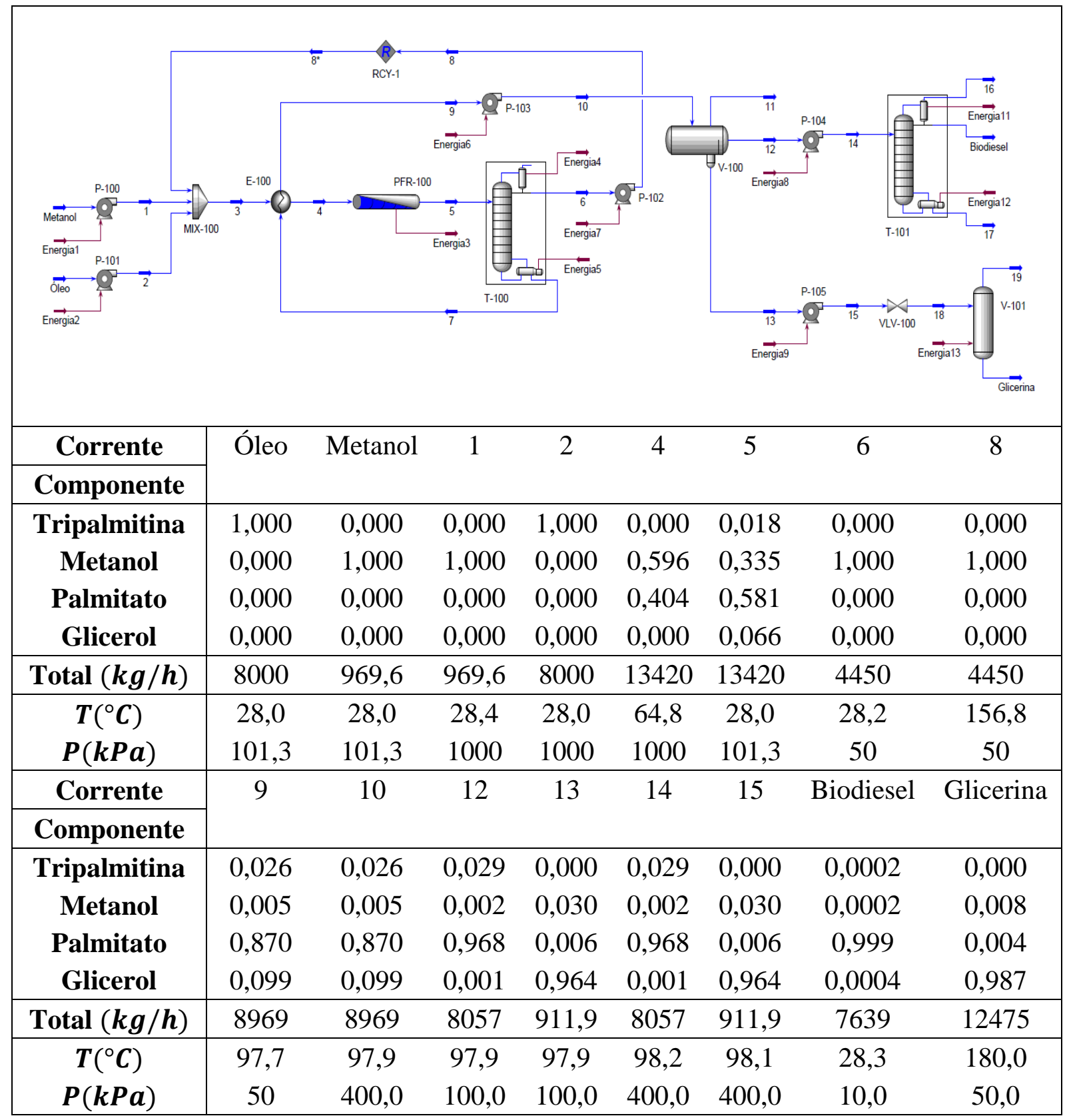

Figura 1 - Diagrama de Fluxo de Processo (PFD) da unidade. 
modo isotérmico a $64,8^{\circ} \mathrm{C}$. A corrente 5 como efluente do reator foi enviada para a coluna de destilação T-100 com 5 estágios teóricos para reciclar o álcool em excesso até o MIX-100. Esta coluna foi operada a vácuo, com uma pressão absoluta de $50 \mathrm{kPa}$ para evitar degradação térmica dos componentes (Zhang et al., 2003). A corrente 7 aquecida do fundo coluna foi utilizada para a troca térmica com a corrente 3 no trocador E-100 configurando-se assim uma integração energética no processo.

A corrente 7, constituída de majoritariamente de palmitato de metila e glicerina, foi bombeada com o auxílio da bomba centrífuga P-103, e enviada para o decantador V-100, no intuito de separar as fases. A corrente 12 de líquido leve, composta majoritariamente de palmitato de metila, foi então bombeada com o auxílio da bomba P-104 até a coluna de destilação T-101, dimensionada com 12 estágios teóricos. Esta coluna, operada também a vácuo para evitar a máxima de temperatura de degradação térmica dos componentes, igual a $250^{\circ} \mathrm{C}$, foi utilizada para purificar o palmitato de metila para formar a corrente Biodiesel, o produto principal do processo. As correntes 16 e 17 foram consideradas como resíduos, mas com baixas vazões mássicas. A corrente 13 de glicerina como líquido pesado foi bombeada com o auxílio de P-105 e enviada para o flash a vácuo V-101, onde foi obtida a corrente de Glicerina purificada.

Na Figura 1, o biodiesel obtido da coluna teve uma pureza de 99,9\% m/m, estando este valor em acordo com as especificações da ANP. Adicionalmente, a corrente de saída de glicerina possuiu uma pureza de $98,7 \% \mathrm{~m} / \mathrm{m}$, com maior valor agregado que o obtido pela rota homogênea. Este valor de pureza concordou portanto com o trabalho de Di Serio et al. (2008), que apontou um valor mínimo de $98 \% \mathrm{~m} / \mathrm{m}$. Esta glicerina purificada poderá compensar uma parte dos custos do processo, que em geral são elevados em virtude do custo da matéria prima.

Os resultados dos projetos dos equipamentos, realizados a partir dos resultados da simulação, estão mostrados na Tabela 2.

O trocador de calor E-100 foi especificado como sendo da classe AES, segundo a norma TEMA. Esta classe de trocador possui um cabeçote flutuante, que é ideal para um intervalo de temperatura maior do $50^{\circ} \mathrm{C}$, como é o caso em questão, evitando assim problemas relacionados a tensões térmicas elevadas no casco (Towler; Sinnot, 2008). Os pratos valvulados foram escolhidos para as colunas de destilação em virtude das baixas perdas de carga em cada prato requeridas para colunas do operando a vácuo, com o mínimo de gotejamento entre os mesmos. 
Tabela 2 - Dimensões e condições operação dos equipamentos de processo

\begin{tabular}{|c|c|c|c|}
\hline Equipamento & Identificador & Dimensões e Condições de Operação & Valores \\
\hline \multirow[t]{5}{*}{ Trocador de Calor } & E -100 & $\phi_{c}(m)$ : diâmetro do casco & 0,54 \\
\hline & & $\phi_{t}(m)$ : diâmetro dos tubos & 0,019 \\
\hline & & $L_{t}(m)$ : comprimento dos tubos & 2,44 \\
\hline & & $N_{t}:$ número de tubos & 356 \\
\hline & & Classe na norma TEMA & AES \\
\hline \multirow[t]{4}{*}{ Reator } & PFR-100 & $D_{r}(m)$ : diâmetro interno do reator & 0,30 \\
\hline & & $L_{r}(m)$ : comprimento do reator & 40,0 \\
\hline & & $m_{c}(\mathrm{~kg}):$ massa de catalisador & 3129 \\
\hline & & $Q_{\text {reator }}(k W)$ : carga térmica & 1926 \\
\hline \multirow[t]{8}{*}{ Coluna de Destilação } & $\mathrm{T}-100$ & $N_{e}:$ número de estágios & 5 \\
\hline & & $R:$ razão de refluxo & 0,01 \\
\hline & & Recuperação no produto de topo (\%) & 99 \\
\hline & & $Q_{c}(k W):$ no condensador & 959,1 \\
\hline & & $Q_{r}(k W):$ no refervedor & 1217 \\
\hline & & $D_{t}(m)$ : diâmetro da coluna & 0,914 \\
\hline & & $T S(m)$ : espaçamento entre pratos & 0,610 \\
\hline & & $N_{v}:$ número de válvulas por prato & 69 \\
\hline \multirow[t]{8}{*}{ Coluna de Destilação } & T-102 & $N_{e}:$ número de estágios & 12 \\
\hline & & $R:$ razão de refluxo & 0,09 \\
\hline & & Recuperação no produto de topo (\%) & 0,995 \\
\hline & & $Q_{c}(k W)$ : potência no condensador & 1370 \\
\hline & & $Q_{r}(k W)$ : potência no refervedor & 1408 \\
\hline & & $D_{t}(m)$ : diâmetro da torre & 1,37 \\
\hline & & $T S(m)$ : espaçamento entre pratos & 0,610 \\
\hline & & $N_{v}:$ número de válvulas por prato & 1888 \\
\hline \multirow[t]{3}{*}{ Decantador } & V-100 & $t(h):$ tempo de residência & 0,46 \\
\hline & & $D_{d}(m)$ : diâmetro do decantador & 1,09 \\
\hline & & $L_{d}(m)$ : comprimento do decantador & 5,45 \\
\hline \multirow[t]{3}{*}{ Vaso Flash } & V-102 & $D_{f}(m)$ : diâmetro do Flash & 0,46 \\
\hline & & $H_{f}:$ altura do flash & 2,52 \\
\hline & & $Q_{f}(k W):$ carga térmica & 83,8 \\
\hline \multirow[t]{6}{*}{ Bombas } & $\mathrm{P}-100$ & \multirow{6}{*}{$W_{b}(k W)$ : potência das bombas } & 0,41 \\
\hline & P-101 & & 3,02 \\
\hline & P-102 & & 1,37 \\
\hline & $\mathrm{P}-103$ & & 1,36 \\
\hline & P-104 & & 1,07 \\
\hline & $\mathrm{P}-105$ & & 0,09 \\
\hline
\end{tabular}




\section{CONCLUSÕES}

Baseado nos resultados apresentados neste trabalho, foi possível concluir o seguinte:

- A simulação demonstrou que o catalisador heterogênea utilizado é tecnicamente viável para produção de biodiesel em escala industrial com ótima conversão do óleo de dendê;

- A purificação do biodiesel envolveu etapas menos complexas que na rota homogênea, com o atendimento das especificações da ANP e baixas vazões de resíduos;

- A glicerina produzida no processo apresentou um grau de pureza mais elevado que a rota homogênea, com maior valor agregado.

\section{REFERÊNCIAS}

ASPEN HYSYS. Operation Guide. Burlington, MA, USA: Aspen Technology, 2011.

ATADASHI, I. M.; AROUA, M. K.; AZIZ, A. A. Biodiesel separation and purification: A review. Renewable Energy, v. 36, p. 437-443, 2011.

DI SERIO. M. et al. Heterogeneous Catalysts for Biodiesel Production. Energy \& Fuels, v. 22, p. 207-217, 2008.

LEE, S; POSARAC, D.; ELLIS, N. Process simulation and economic analysis of biodiesel production processes using fresh and waste vegetable oil and supercritical methanol. Chemical Engineering Research and Design, v. 89, p. 2626-2642, 2011.

McCABE, W. L.; SMITH, J. C.; HARRIOTT, P. Unit Operations of Chemical Engineering. New York, USA: Mc Graw Hill, 2005.

MORAIS, S. et al. Simulation and life cycle assessment of process design alternatives for biodiesel production from waste vegetable oils. Cleaner Production, v. 18, p. 12511259, 2010.

SANTANA, G. C. S. Simulação e Análise de Custos na Produção de Biodiesel a Partir de Óleos Vegetais. Campinas, SP: Unicamp, 2008. Originalmente apresentada como tese de doutorado.

TOWLER, G.; SINNOT, R. Chemical Engineering Design: Principles, Practice and Economics of Plant and Process Design. Oxford, UK: Elsevier, 2008.

WEST, A. H.; POSARAC, D.; ELLIS, N. Assessment of four biodiesel production processes using HYSYS.Plant. Bioresource Technology, v. 99, p. 6587-6601, 2008.

XIAO, Y. et al. Kinetics of the Transesterification Reaction Catalyzed by Solid Base. Energy \& Fuels, v. 24, p. 5829-5833, 2010.

XIAO, Y. et al. Experimental and Modeling Study of Continuous Catalytic Transesterification to Biodiesel in a Bench-Scale Fixed-Bed Reactor. Industrial \& Engineering Chemistry Research, v. 51, p. 11860-11865, 2012.

ZHANG, Y. et al. Biodiesel production from waste cooking oil: 1. Process design and technological assessment. Bioresource Technology, v. 89, p. 1-16, 2003. 